

\title{
Matter Diffusion in Hexagonal Columnar Phases
}

\author{
M. Daoud, K. Rä̈os, M. Gharbia, A. Gharbi \\ Groupe des Cristaux Liquides-Ecole Supèrieure des Sciences \\ et Techniques de Tunis-5, Avenue Taha Hussein-BP 56-Tunis 1008-Tunisie. \\ Laboratoire des Cristaux Liquides et des Polymères \\ Faculté des Sciences de Tunis-Campus Universitaire-1002 Tunis-Tunisie. \\ H.T. Nguyen \\ Centre de Recherche Paul Pascal-Avenue A. Schweitzer-33600 Pessac-France.
}

Received 08 September, 1998

\begin{abstract}
Matter diffusion experiments are carried out in hexagonal columnar phases of mesogenic disk-like and polycatenar compounds. The diffusion characteristics of two dyes of different sizes diffused into these mesophases are determined by measuring the diffusion coefficients along the columns and perpendicularly to them. The nature of diffusion versus the structure of these mesophases is discussed in comparison with previous results obtained in nematic and smectic systems.
\end{abstract}

PACS numbers: 61.30.v; 62.20.Fe; 66.30.J.

\section{Introduction}

It is well-established that the hexagonal columnar phases can be exhibited by thermotropic and lyotropic mesogenic compounds which consist of disk-like rodlike or amphiphilic molecules.

In recent years $\Gamma$ several experimental and theoretical studies have been carried out on thermotropic hexagonal columnar phases such as: X-ray investigationГRayleigh scattering from column ondulationsГcolumn buckling instability $\Gamma$ point defects $\Gamma$ surface tension and elasticity [1-12] etc.... These studies have shown that the columnar behaviour depends on the nature $\Gamma$ the shape and the stacking mode of the mesogenic molecules in columns. In addition $\Gamma$ some typical coefficients have been measured $\Gamma$ such as the elastic curvature $\Gamma$ compressibility modulus $\Gamma$ penetration length $\Gamma$ elastic shear modulus etc. However $\Gamma$ the diffusion coefficients have not been determined $\Gamma$ except for an indirect estimation [3] Talthough the first investigation of diffusion in mesophases was realized by T. Svedberg since 1918 [13] and this phenomenon had been widely studied in nematic and smectic mesophases during the last thirty years [14]. Thus $\Gamma$ several techniques have been used. Among them are direct measurements of Mass Transport [15-17] $\Gamma$ Nuclear Magnetic Resonance methods [18-20] $\Gamma$ Quasi-Elastic Neutron Scattering [21L22] etc....

More recently $\Gamma$ we have studied the diffusion phenomenon in thermotropic hexagonal columnar mesophases. We have reported the first measurements of diffusion coefficients and anisotropic ratios of some discotic and phasmidic mesogens [23-26]. Experimentally $\Gamma$ we have used an optical absorption technique which consists in dissolving appropriate probes through the mesophases. The diffusion is proved by direct measurements of the absorbed light by the sample or by taking pictures of the sample versus diffusion time. A mirage effect technique is also used. In both cases the diffusion coefficients are measured in two geometries $\Gamma$ along the columns $\left(D_{\|}\right)$and perpendicularly to them $\left(D_{\perp}\right)$. Then Tanisotropic ratios $D_{\|} / D_{\perp}$ are deduced.

All experimental values are discussed. A comparison between the diffusion coefficients and anisotropic ratios to the results obtained in nematic and smectic

\footnotetext{
${ }^{*}$ Author for correspondence.
} 
mesophases [14] may lead to a better understanding of the relationship between the mesomorphic properties and the molecular structure of these mesophases.

\section{Description of thermotro- pic hexagonal columnar mesophases}

It is well-known that beyond the classical liquid crystals $\Gamma$ i.e. nematic $\Gamma$ cholesteric and different lamellar phases such as $S_{A}, \quad S_{C}, \quad S_{B}, \quad S_{F}$ etc.... which consist of rod-like molecules [27] $\Gamma$ thermotropic columnar mesophases have been observed by S. Chandrasekhar et al since 1977 [28] with a discotic mesogen $\Gamma$ the hexa-n-alcanoate of benzene. Later $\Gamma$ several discotic mesogenic compounds possessing alkoxy $\Gamma$ alkanoyloxy chains with different nature of the core and exhibiting these new types of mesophases have been synthesized [29134] such the hexa-n-alkoxytriphenylenes (HET) $\Gamma$ the hexa-n- alkanoyloxytriphenylenes (HAT) $\Gamma$ the hexa-n-alkanoyloxybenzenes (HAB) $\Gamma$ the hexa-nalkoxybenzoates of triphenylene (HBT) $\Gamma$ the hexa-nalkanoyloxytruxenes (HATX) etc.... Structural studies by X-ray diffractionTrealized by A.M. Levelut [4] have shown that these mesophases consist of a regular packing of parallel and independent columns of molecules. In the columns the disk-like molecules which pile up are staked and arranged in a two-dimensional network (Fig. 1). In addition $\Gamma$ the rigid cores of the disk-like molecules are normal or tilted with respect to the columnar axis. According to the symmetries of these mesophases $\Gamma$ the lattices are called $D_{h}$ (hexagonal) $\Gamma D_{o b}$ (oblique) $\Gamma D_{r}$ (rectangular)) etc....

The columnar structure of these mesophases have been characterizated by other techniques such NMR spectroscopy $\Gamma$ Mass Transport etc.... Also $\Gamma$ using optical texture observations [31] $\Gamma$ one can find large homeotropic domains revealing the uniaxial nature of these mesophases. Furthermore $\Gamma$ little homeotropic hexagons which reveal an hexagonal lattice were observed.

Soon afterwards Tother homologous series of mesogenic compounds which also exhibit thermotropic hexagonal columnar mesophases have been synthesized. Among them we can remind some compounds:

(i) Chiral molecules $\Gamma$ i.e. propellar-like molecules which rotate in different directions within their columns without destroying the columnar structure. The nor- mals to disk-like molecules are constrained to lie parallel to columnar axes [35-37]. Several experimental studies and theoretical models describing the columnar mesophases exhibited by these molecules are realized [38-40].

(ii) Polycatenar mesogensTi.e. molecules with a long rod-like rigid core ending in two half disk moities and three $\Gamma$ four $\Gamma$ five or six aliphatic chains in ortho $\Gamma$ para and meta positions are grafted at each end (Fig. 2aIb) [41-46]. The hexagonal columnar mesophases observed in this case have the same symmetry as the disc-like molecules. Furthermore the columns are shaped with slices containing three $\Gamma$ four or five molecules (Fig. 2c). Some types of these compounds will be studied in the following sections.

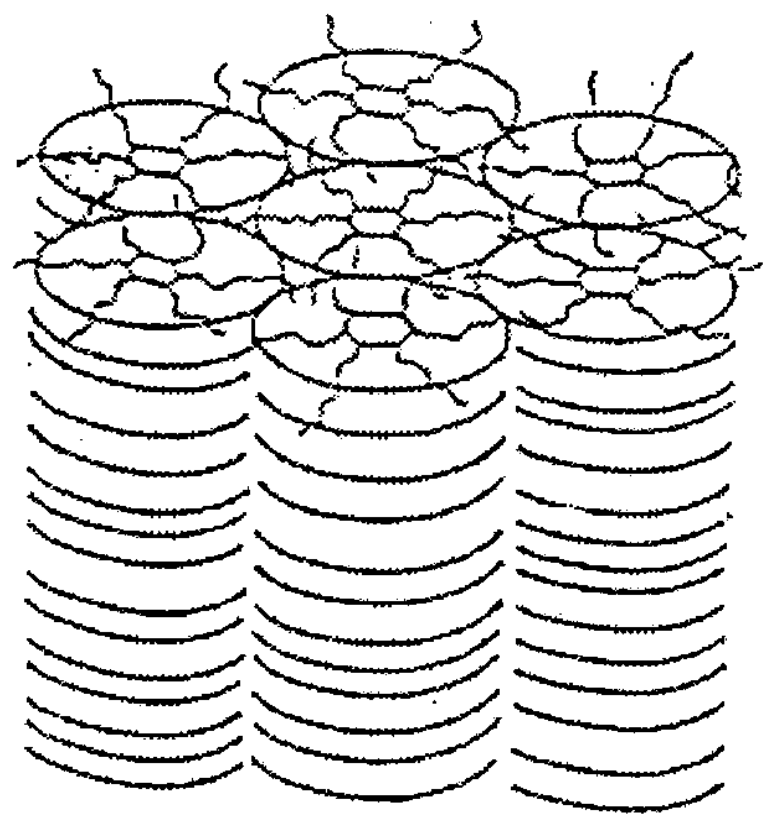

Figure 1. Schematic representation of the structure of the columnar discotic liquid crystal.

(iii) Heteroatomic salts in which the central core part consists of a polarisable heteroatomic cation. These salts exhibit $\Gamma$ particularly $\Gamma$ an ordered columnar mesophase from room temperature to $200^{\circ} \mathrm{C}$. Furthermore $\Gamma$ the columnar slice differ from the discotic and polycatenar ones. Several studies have shown that the number $\Gamma$ the position $\Gamma$ the length of the flexible chains and the nature of the anions are at the origin of the polymorphism properties [47-49].

One should note that hexagonal columnar mesophases may be also exhibited by many lyotropic liquid crystals which consist of amphiphilic molecules 
in presence of water. These molecules are able to build up structures exhibiting a long range crystalline order $\Gamma$ although they are locally disordered in a liquid-like along the columnar axis [50151]. The first lyotropic columnar mesophases were observed by A. Skoulios et al [52]. As for example $\Gamma$ we note the liquid crystalline phtalocyanines which are prepared for the first time about fifteen years ago [53]. These amphiphilic compounds with alkyl chains at their peripheral positions (Fig. 3a) display lyotropic mesophases in which the crown ether rings form large columnar aggregates in solution. Two types of lyotropic columnar mesophases are identified. The first one corresponds to cylinders with a paraffinic core surrounded by matter $\Gamma$ whereas in the second type $\Gamma$ the core of each cylinder contains water surrounded by the amphiphilic molecules (Fig. $3 \mathrm{~b} \Gamma \mathrm{c})$.
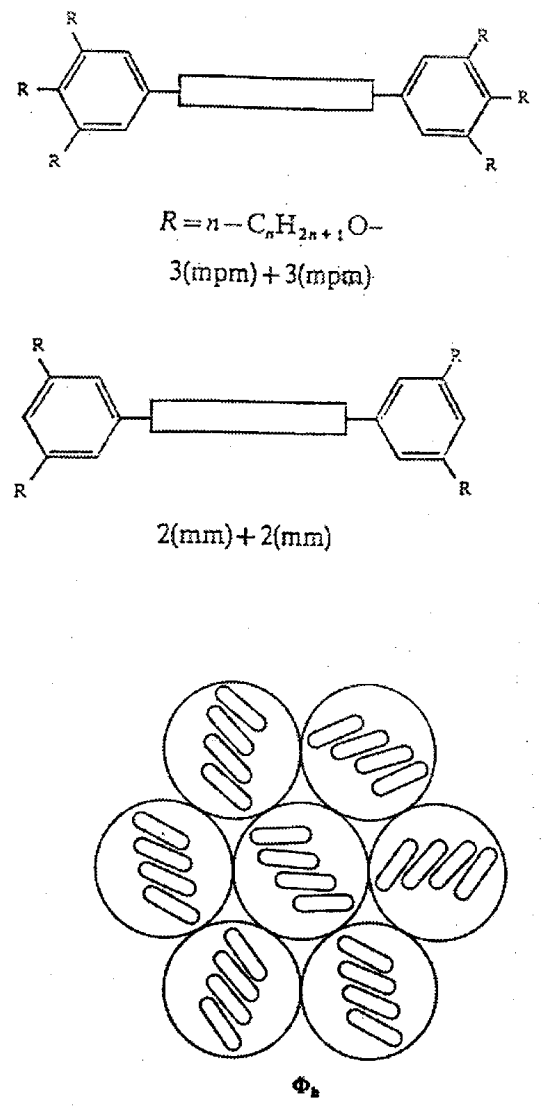

(a)

(b)

(c)

Figure 2. Schematic representation of two types of polycatenar molecules (a) an hexacatenar, called "phasmidic" molecule (b) a tetracatenar, called "biforked" molecule. (c) schematic representation of a slice of the polycatenar columnar phase (from Ref. 44,55).

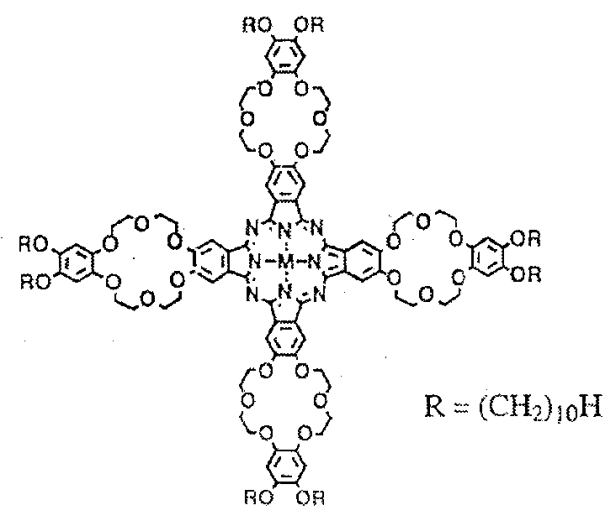

(a)

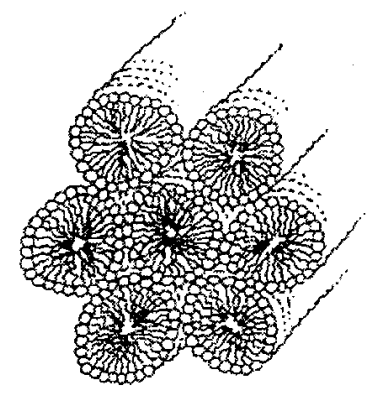

(b)

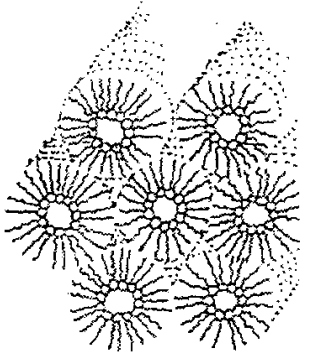

(c)
Figure 3. Schematic representation of lyotropic columnar phases: (a) chemical formulas of phtalocyanine molecule; (b) direct columnar phase; (c) inverse columnar phase (from Ref 49).

\section{Bulk and surface diffusion in thermotropic hexagonal columnar mesophases}

\section{III.1 Material}

\section{a) Disk-like compounds}

The disk-like molecules are formed by a rigid aromatic core surrounded by six aliphatic chains which are necessary to exhibit columnar mesophases [29-34]. Experiments are performed with the four following mesogenic disk-like compounds:

* Three hexa-n-alkoxytriphenylenes $\left(C_{n} H E T\right)$ : the hexa-n-pentyloxytriphenylene $\left(C_{5} H E T\right)$, the hexan-hexyloxytriphenylene $\left(C_{6} H E T\right)$ and the hexa-noctyloxytriphenylene $\left(\mathrm{C}_{8} \mathrm{HET}\right)$. These molecules consist of triphenylene cores surrounded by six alkoxy 
chains $\left(R=0-C_{n} H_{2 n+1}\right)$. We present in figure (4a) the chemical formulas of these compounds. All these compounds exhibit a thermotropic hexagonal columnar mesophase $\left(D_{h}\right)$ as follow $\Gamma$

$$
\begin{aligned}
& \left(C_{5} H E T\right) \quad K \stackrel{69^{\circ} \mathrm{C}}{\longrightarrow} D_{h} \stackrel{122^{\circ} \mathrm{C}}{\longrightarrow} I \\
& \left(C_{6} H E T\right) \quad K \stackrel{68^{\circ} C}{\longrightarrow} D_{h} \stackrel{97^{\circ} C}{\longrightarrow} I \\
& \left(C_{8} H E T\right) \quad K \stackrel{66.8^{\circ} \mathrm{C}}{\longrightarrow} D_{h} \stackrel{85.6^{\circ} \mathrm{C}}{\longrightarrow} I
\end{aligned}
$$

* One hexa-n-alkanoyloxytriphenylene $\left(C_{n} H A T\right)$ : this type of molecule has the same core that the last ones but with six alkanoyl lateral chains $(R=$ $\left.-O C O-C_{n} H_{2 n+1}\right)$. The compound used is the hexan-dodecanoate of triphenylene $\left(C_{11} H A T\right)$ (Fig. $\left.4 \mathrm{~b}\right)$. lt exhibits three stable mesophases between $80^{\circ} \mathrm{C}$ and $122^{\circ} \mathrm{C}$.

$$
\left(C_{11} H A T\right) K \stackrel{80^{\circ} \mathrm{C}}{\longrightarrow} D_{h o} \stackrel{93^{\circ} \mathrm{C}}{\longrightarrow} D_{r} \stackrel{111^{\circ} \mathrm{C}}{\longrightarrow} D_{h d} \stackrel{122.3^{\circ} \mathrm{C}}{\longrightarrow} I
$$

$D_{h}, D_{r}$ and $D_{h d}$ correspond $\Gamma$ respectively $\Gamma$ to the ordered hexagonal $\Gamma$ rectangular and disordered hexagonal phases. The choice of these samples allows us to study the effects of the length $\mathrm{the}$ shape of the chains bound to the triphenylene disks and the stacking order of disks in columns.
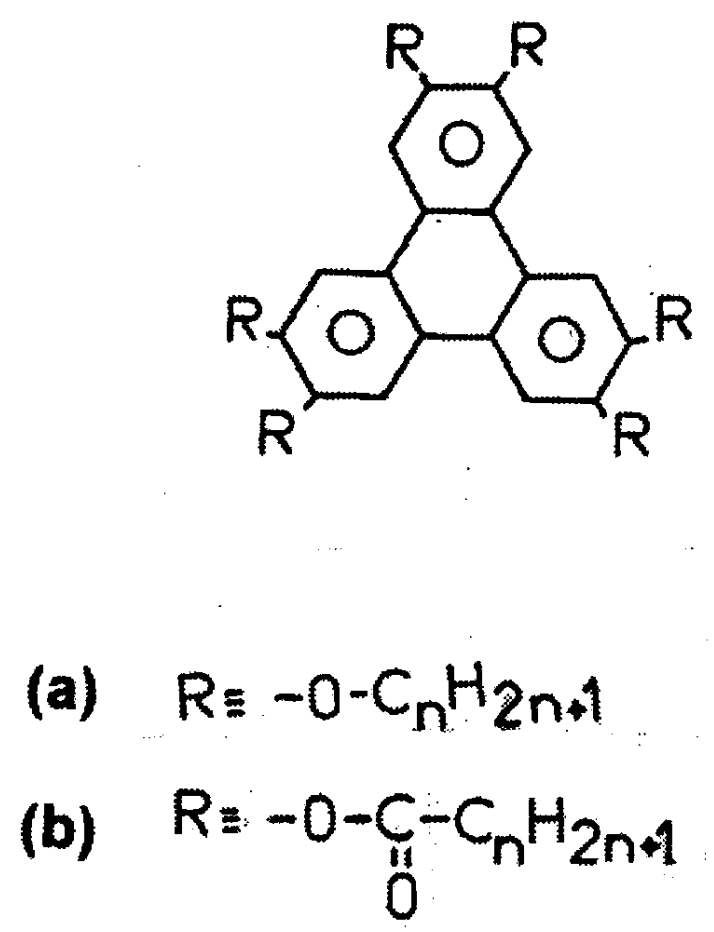

Figure 4. Chemical formulas of triphenylene compounds, (a) the hexa-n-alkoxytriphenylenes $\left(C_{n} H E T\right)$ : (b) the hexa-nalkanoyloxytriphenylene $\left(C_{n} H A T\right)$ :

\section{b) Polycatenar compounds}

The polycatenar compounds present a clear rod-like core which is composed of an elongated polyaromatic part containing $\Gamma$ in general $\Gamma$ five rings connected in the para position and normal aliphatic chains grafted only on two terminal benzene rings $\Gamma$ in the para and meta positions. Furthermore $\mathrm{unlike}$ the discotic mesogens where the disks are staked one on top the other to form liquid-like columns $\Gamma$ in the polycatenar mesogens the slice of a column is made by a cluster of rigid cores arranged side by side and surrounded by aliphatic chains. Each slice is formed by three molecules for the hexacatenar and four ones for the tetracatenar mesogens. Taking into account the number of aliphatic chains and the possible combination of their enchoring points into meta and para positions with regard to the linking group of the central core $\Gamma$ a nomenclature is proposed. So $\Gamma$ the compounds with six $\Gamma$ five $\Gamma$ four or three chains are named respectively the hexaГpentaГtetra and three catenars [42-46]. Some studies have shown that the molecular structure of polycatenar compounds holds an intermediate position between mesogenic disks and mesogenic rods. Furthermore $\mathrm{Tthe}$ polymesomorphism is very similar to that of lyotropic systems [50].

Experiments are performed with three polycatenar compounds with five and six-benzene-ring cores. They differ also by the length $\Gamma$ number $\Gamma$ nature and the position of aliphatic chains bound on the core. In these materials Tonly these columnar mesophases having the same symmetry as the disk-like mesogens are considered.

* One hexacatenar mesogen (called "Phasmid" from the name of six legged stick-like insects). This type of polycatenar mesogen has three alkyloxy chains $R=$ $-0-C_{n} H_{2 n+1},(n=12)$ in the meta $\Gamma$ para and meta position at each end $[3(\mathrm{mpm})+3(\mathrm{mpm})]$ (figure $5 \mathrm{a})$. lt undergoes the following phase transitions:

$$
K \stackrel{70^{\circ} C}{\longrightarrow} \Phi_{o b} \stackrel{81.5^{\circ} C}{\longrightarrow} \Phi_{h} \stackrel{92^{\circ} C}{\longrightarrow} I
$$

$\Phi_{o b}$ and $\Phi_{h}$ mean phasmidic mesophases with respectively an oblique and hexagonal two dimensional lattice. The existence of stability of columnar mesophases depend on the flexibility or rigidity of the central group. The term "phasmidic" phases (denoted $\Phi \Gamma$ the initial letter of the Greek root) is used to distinguish this new type of columnar mesophases which differs from disklike mesogens in its molecular organisation. 


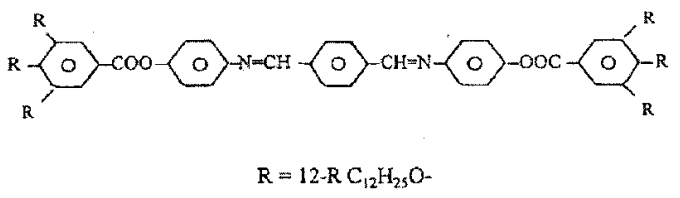

(a)

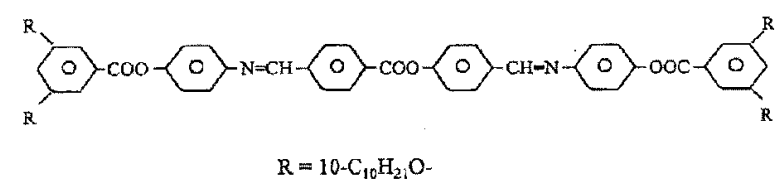

(b)

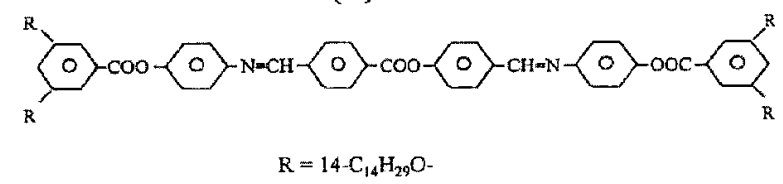

(c)

Figure 5. Chemical formulas of polycatenar molecules: (a) an hexacatenar $(\mathrm{n}=12)$; (b) a tetracatenar $(\mathrm{n}=10)$; (c) a tetracatenar $(\mathrm{n}=14)$.

* Two tetracatenar mesogens (called "biforked" mesogens). This type of molecule has two different alkyloxy chains $R=-0-C_{n} H_{2 n+1}$ (we have used $n=10$ and $n=14)$ connected in the meta meta position at each end $[2(m m)+2(m m)]$ (figure $5 \mathrm{~b} \Gamma)$. They respectively exhibit the following mesophases:

$$
\begin{aligned}
& K \stackrel{76^{\circ} C}{\longrightarrow} \Phi_{h} \stackrel{110^{\circ} C}{\longrightarrow} I \\
& K \stackrel{66^{\circ} C}{\longrightarrow} \Phi_{h} \stackrel{118^{\circ} C}{\longrightarrow} I
\end{aligned}
$$

The choice of these compounds has been made because they all present thermotropic hexagonal mesophases. This allows to compare the results to the discotic liquid crystal ones. In addition $\Gamma$ the different number $\Gamma$ position and length of the aliphatic chains allow to examine the influence of these parameters on the anisotropic diffusion ratios $D_{\|} / D_{\perp}$.

\section{c) lmpurities}

The impurities diffused in these mesophases are appropriate biological dyes with different molecule sizes. Two have been chosen from several dyes: a red dye $\Gamma$ called Red Oil OГthe 1-[4(xylylazo)xylylazo]-2-Naphtol with a molecular diameter $0.75 \mathrm{~nm}$ and a yellow one the 4-Phenylazophenol with a molecular diameter $0.45 \mathrm{~nm}$. These dyes present melting points beyond the temperature domain of the studied liquid crystals $\left(\operatorname{mp}_{\text {red dye }}=120^{\circ} \mathrm{C}\right.$ and $\left.m p_{\text {yellow dye }}=156^{\circ} \mathrm{C}\right) . . \ln$ addition $\Gamma$ experimentally $\Gamma$ we do not observe a transition of the liquid crystal-dye mixture to the isotropic phase at temperatures $<90^{\circ} \mathrm{C}$ for the red dye and at temperatures $<115^{\circ} \mathrm{C}$ for the yellow one. This allows to detect the dye concentration diffused into the mesophase versus the time and the position. The chemical formulas of these dyes are presented in figure $(6 \mathrm{a} \Gamma \mathrm{b})$.

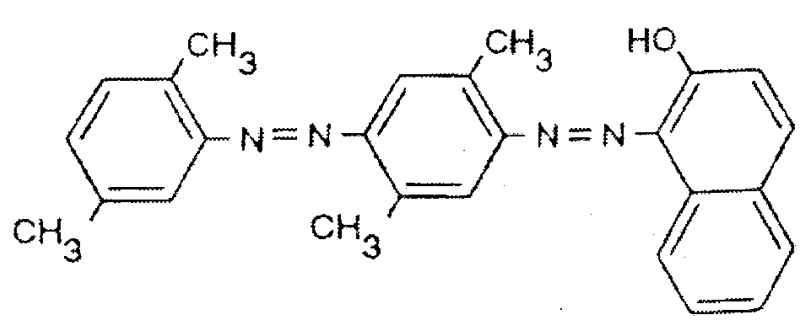

(a)

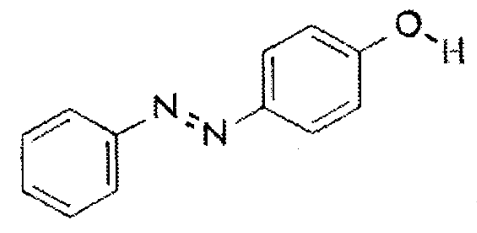

(b)

Figure 6. Chemical formulas of impurity molecules: (a) the 1-[4-(xylylazo)xylylazo]-2-Naphtol (red dye); (b) the 4phenylazophenol (yellow dye).

\section{III.2 Theory}

It is well-known that the main property of mesophases $\Gamma$ which distinguish them from both $\Gamma$ isotropic liquids and cristalline solids $\Gamma$ is the order parameter $\mathrm{S}$ which is described by the following equation:

$$
S=\frac{1}{2}\left\langle 3 \cos ^{2} \theta-1\right\rangle
$$

Where $\theta$ is the angle between the long molecular axis and the director $n$.

In general $\Gamma$ the diffusion coefficient of mesophases will be a second rank tensor $D$, the symmetry of which is related to the symmetry of mesophase. $D$ depends 
on the order parameter and hence its components can be written by [14]:

$$
\begin{gathered}
D_{\perp}=<D>(1-S)+S D_{\perp}^{0} \\
D_{\|}=<D>(1-S)+S D_{\|}^{0}
\end{gathered}
$$

where $\langle D\rangle$ Toften called average diffusion coefficient $\Gamma$ is given by:

$$
<D>=<D^{0}>=\frac{1}{3}\left(2 D_{\perp}^{0}+D_{\|}^{0}\right)=\frac{1}{3}\left(2 D_{\perp}+D_{\|}\right)
$$

$D^{0}$ is the diffusion coefficient tensor of a perfectly ordered phase $\Gamma D_{\|}$and $D_{\perp}$, are the diffusion coefficients $\Gamma$ respectively parallel to the director $n$ and perpendicular to it.

As in the fluid systems $\Gamma$ the relationship between the diffusion coefficient and the impurity concentration which diffuses in the mesophases can be described by the Fick equation.

For $1 \mathrm{D}$ treatment $\Gamma C(x, t)$ is related to the diffusion coefficient $D$ by:

$$
\frac{\partial C(x, t)}{\partial t}=D \frac{\partial^{2} C(x, t)}{\partial x^{2}}-<\Gamma_{0}>C(x, t)
$$

$<\Gamma_{0}>C(x, t)$ represents the transverse diffusion and $\Gamma_{0}$ is the jump frequency. Equation (1) agrees with the following solution $\Gamma$

$$
C(x, t)=C(0, t) \exp \left(-\frac{x^{2}}{4 D t}\right)
$$

Furthermore $\Gamma$ the concentration at the origin $\mathrm{C}(0 \mathrm{It})$ is given by:

$$
C(0, t)=\frac{Q}{\sqrt{\pi D t}} \exp \left(-<\Gamma_{0}>t\right)
$$

Where $Q$ represents the amount of impurity diffused by unit of area such as:

$$
Q=\int_{0}^{\infty} C(x, t) d x
$$

Equations (2) and (3) predict that $\mathrm{C}(\mathrm{xIt})$ is described by half a gaussian curve centered on $C(O, t)$ which decreases as $\sqrt{t}$ with an attenuation factor $\exp \left(-<\Gamma_{0}\right\rangle$ $t)$.

From the gaussian curve $\Gamma$ one can measure the gaps $A \propto \sqrt{D_{\|} t}$ (along the columns) and $B \propto \sqrt{D_{\perp} t}$ (perpendicularly to them). Indeed $\Gamma$ in planar orientation $\Gamma$ we deduce that the diffusion in the two perpendicular directions can be described by ellipsoid axes such as:

$$
\frac{X^{2}}{A^{2}}+\frac{Y^{2}}{B^{2}}=1
$$

From these equations $\Gamma$ we can derive the ellipticity for many times:

$$
\frac{A}{B}=\sqrt{\frac{D_{\|}}{D_{\perp}}}
$$

Then $\Gamma$ we can deduce the anisotropic ratio $D_{\|} / D_{\perp}$ from the densitometry of pictures.

\section{III.3 Experiments}

\section{a) Bulk diffusion}

For the bulk diffusion in disk-like mesogens $\Gamma$ the experimental set-up is composed of a spectral lampГa system of lens $\Gamma$ a sample holder placed in a thermostated cell $\Gamma$ a detector and a recorder. The sample is placed between two glass plates treated with mellitic acid as a surfactant. The thickness of the sample can be adjusted with mica spacers from 5 to $80 \mu \mathrm{m}$. Then $\Gamma$ it is oriented in two geometries: columns perpendicular to the plates (homeotropic orientation) and parallel to them (planar orientation). For the lower thickness $\Gamma$ we can observe a good homeotropic orientation when the sample is cooled very slowly $\left(0.05^{\circ} \mathrm{C}\right.$ per min) from the isotropic to the liquid crystal phase. Thus $\Gamma$ we obtain a sample with grains of about $1 \mathrm{~mm}$ of size. The planar orientation is obtained from homeotropic oriented sample to which we apply on the upper plate shearings which amplitude is about $10 \mu \mathrm{m}$. The sample is kept at rest between successive shearings during $10 \mathrm{mn}$ for annealing.

For the dye diffusion experiments $\mathrm{Ta}$ thin film of the impurity is carefully placed on the edge of the lower glass plate $\Gamma$ in contact with the liquid crystal. Some time later $\Gamma$ a concentration gradient of the diffused impurity molecules can be directly visualized with an optical polarizing microscope.

We used an optical absorption technique which consisted principally of detecting the concentration gradient of dye into mesophase due to diffusion. The measurement of the dye concentration variation versus different positions into the sample allows for the measurement of the diffusion coefficients. ActuallyГfrom a light beam propagated through the sample $\Gamma$ we deduce the 
light intensity $\left(l_{a}\right)$ absorbed by impurity. The transmitted light $\left(I_{t}\right)$ is detected by a photodetector. The intensity $(I)$ in the pure sample allows to deduce the absorbed one $l_{a}=I-I_{t}$ which is proportionnel to the concentration of impurity diffusing inside the liquid crystal. QuantitativelyCto determine this concentration $\Gamma$ we measured the diffusion profile versus the position $\mathrm{x}$ and versus the column orientation $\Gamma$ for many times.

For the polycatenar compounds 5 the dye concentration diffused into the mesophase is followed by taking pictures of the mesophase after different times. The sample is placed between two glass plates $L_{1} \Gamma L_{2}$ with a thickness $e=20 \mu \mathrm{m}$. The upper glass contains in the center a circular hole which $\Phi$ is about $1 \mathrm{~mm} \Gamma$ aimed to place the diffusing dyes in contact with the liquid crystal. Thus $\Gamma$ we take several pictures from which we measure the diffusion profile versus the dye position $\Gamma$ the diffusion time and versus the column orientations. The study of these pictures shows that the diffusion symmetry is circular for the homeotropic orientation (Fig. 7a) and elliptical for the planar one (Fig. 7b). The mean ellipse axes correspond to a dye diffusion into the mesophase along the columns and perpendicularly to them. The measurements of the lengths of the ellipse axes allow to deduce the ratio of the diffusion coefficients.

\section{b) Surface diffusion}

The surface diffusion is made in discotic liquid crystal threads which are prepared with the same procedure used by V. Winckle and N.A. Clark [5455]. The used compound is the hexa-n-pentyloxytriphenylene $\left(C_{5} H E T\right)$. The sample is melted between two glass plates of thickness $100 \mu \mathrm{m}$. Then $\Gamma$ the temperature is lowered to a fixed value in the hexagonal phase. When the plates are slowly taken away from each otherГa discotic drop is formed which will give rise to a thread. The motion of the plates is frequently interrupted with a 10 min annealing between each displacement. We obtain quasi-cylindrical threads attached to the glass plates by larger parts $\Gamma$ called "bases" (Fig. 8). Several threads are prepared with different diameters in the range of $10 \mu \mathrm{m}$ to $20 \mu \mathrm{m}$ and lengths in the range of $100 \mu \mathrm{m}$ to $2 \mathrm{~mm}$. We can verify that for the used thickest threads $d(10 \mu m<d<20 \mu m) \Gamma$ the optical axis is oriented along the thread axis; this corresponds to the direction of columns. Before experimenting $\Gamma$ the thread is subjected to a two-hour annealing at the same temperature.
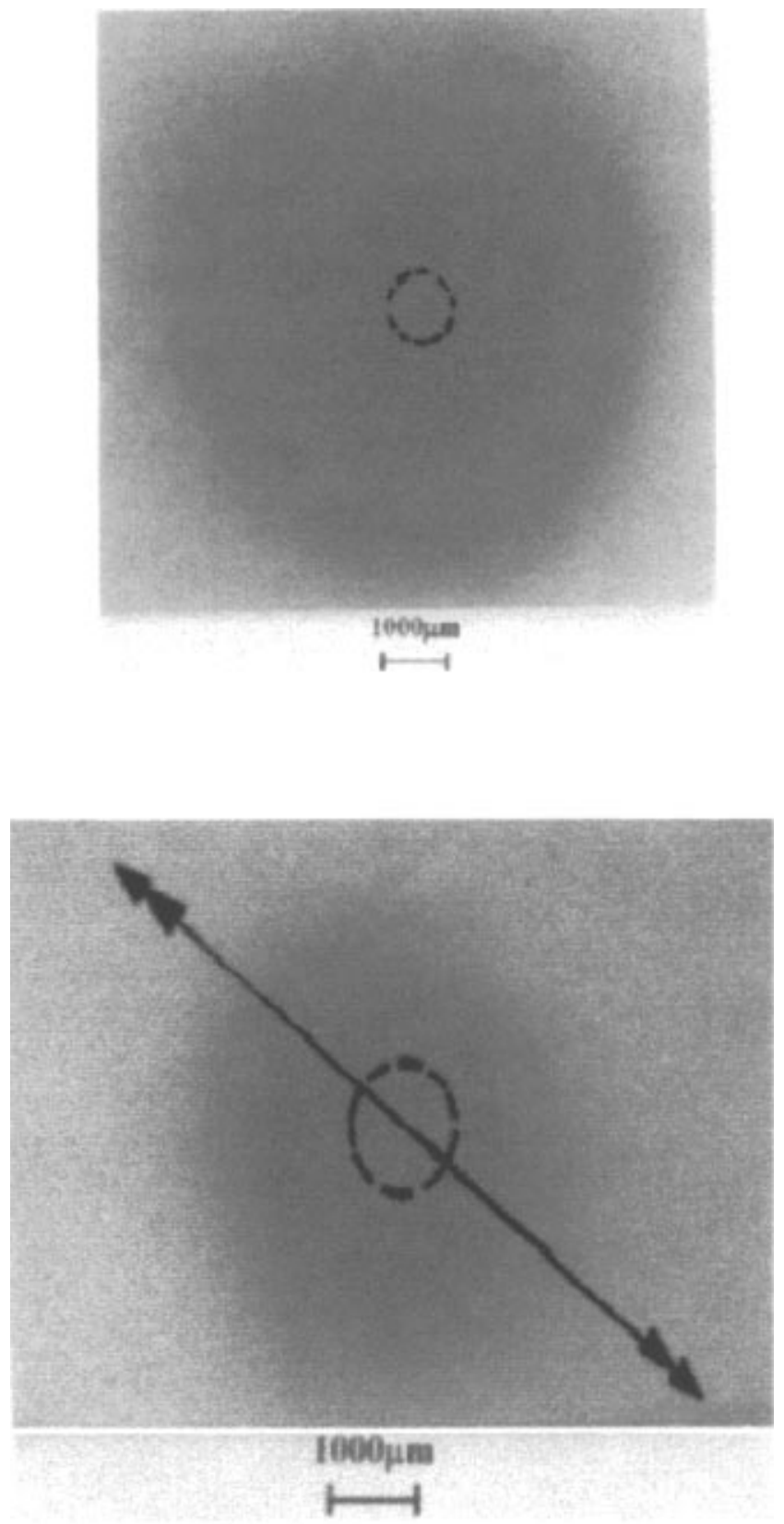

Figure 7. Pictures of red dye concentration profiles of hexacatenar columnar phases: (a) in homeotropic orientation; (b) in planar orientation.

The surface diffusion experiments are performed as follow:

One thread of diameter $\mathrm{d}$ is explored by a Cd spectral lamp rectangular beam of about $40 \mu \mathrm{m} \times 4 \mathrm{~mm}$. The transmitted light is focused by a lens system on a detector. To measure the concentration of yellow dye $\Gamma$ 
the 4-phenylazophenol $\Gamma$ diffused in the thread $\Gamma$ we use the optical absorption technique described in section III-3-a. Actually $\Gamma$ from the light intensity transmitted by the sample for different positions of the photocell along the transmitted rectangular beam we deduce the values of concentration $C(x, t)$ versus $x$. However $\Gamma$ this technique helps to keep the oven with the sample inside in a fixed position $\Gamma$ we only move the photocell over an image Cfive times the size of the transmitted beam.

\section{III.4 Results and discussion}

\section{a) Diffusion coefficients}

In order to measure the diffusion coefficients $D_{\|}$and $D_{\perp}$ of dyes in all studied mesophases $\Gamma$ we plot the variation of the concentration $C(x, t)$ versus the position $x$ for many times. For the bulk diffusion $\Gamma$ we report in figure (9 аІЪГ) $\Gamma$ for example $\Gamma$ the case of $C_{6} H E T$ at $85^{\circ} \mathrm{C}$ for two times and two dyes. We note that half of the gaussian curves are in agreement with Equation (2). They lead to a predictible result i.e. the matter diffusion in the hexagonal columnar phases can be decribed by the Fick Law.

Furthermore $\Gamma$ the plot of $\ln (C(0, t) / C(x, t))$ versus $x^{2}$ for a given time $(t=72 h o u r s)$ shows a linear behaviour (Fig. 10 aIb $)$ ). From the slopes $\Gamma$ one can deduce the diffusion coefficients $D_{\|} \Gamma D_{\perp}$ and $\Gamma$ consequently $\Gamma$ the anisotropic ratios $D_{\|} / D_{\perp}$. All experimental results are presented in table 1.

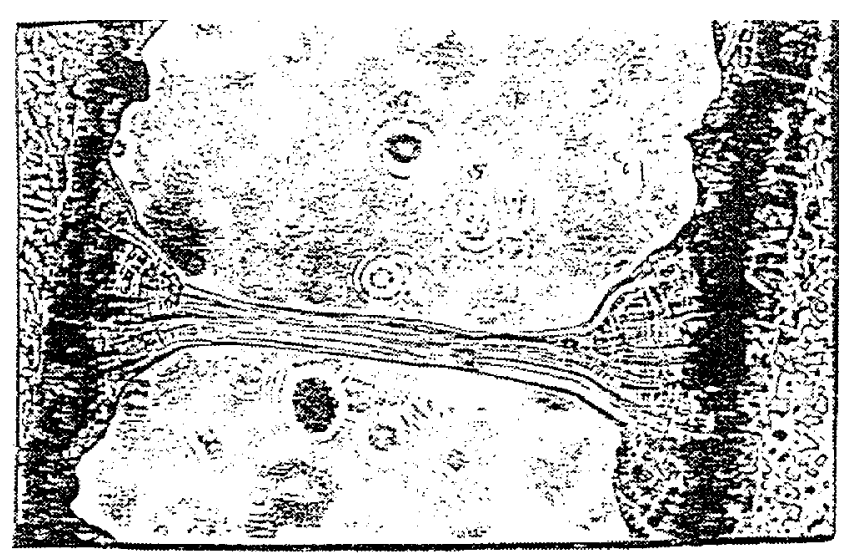

Figure 8. Microscopic view of a thread.
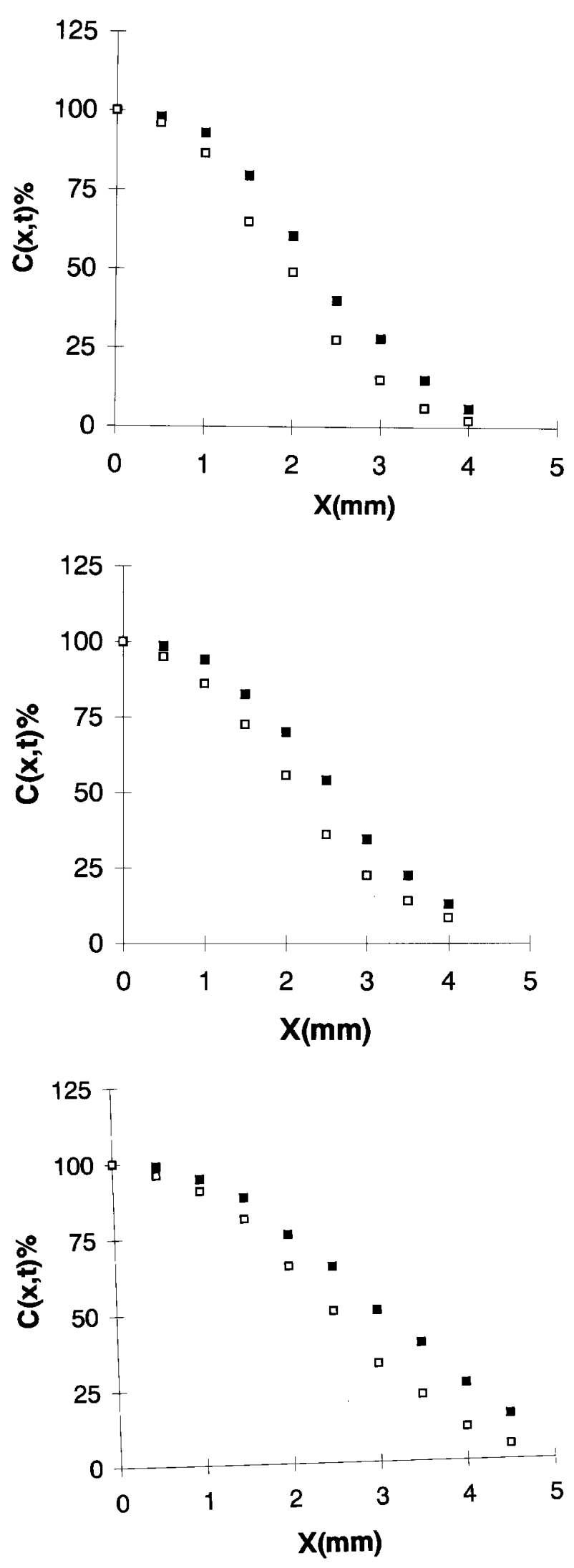

Figure 9. Plot of the dye concentration $\mathrm{C}(\mathrm{x}, \mathrm{t})$ versus position $\mathrm{x}$ of $\left(C_{6} H E T\right)$ at $85^{\circ} \mathrm{C} \mathrm{t} 1=48 \mathrm{~h}, \mathrm{n} \mathrm{t} 2=72 \mathrm{~h}$. (a) in homeotropic orientation (red dye); (b) in planar orientation (red dye); (c) in planar orientation (yellow dye). 

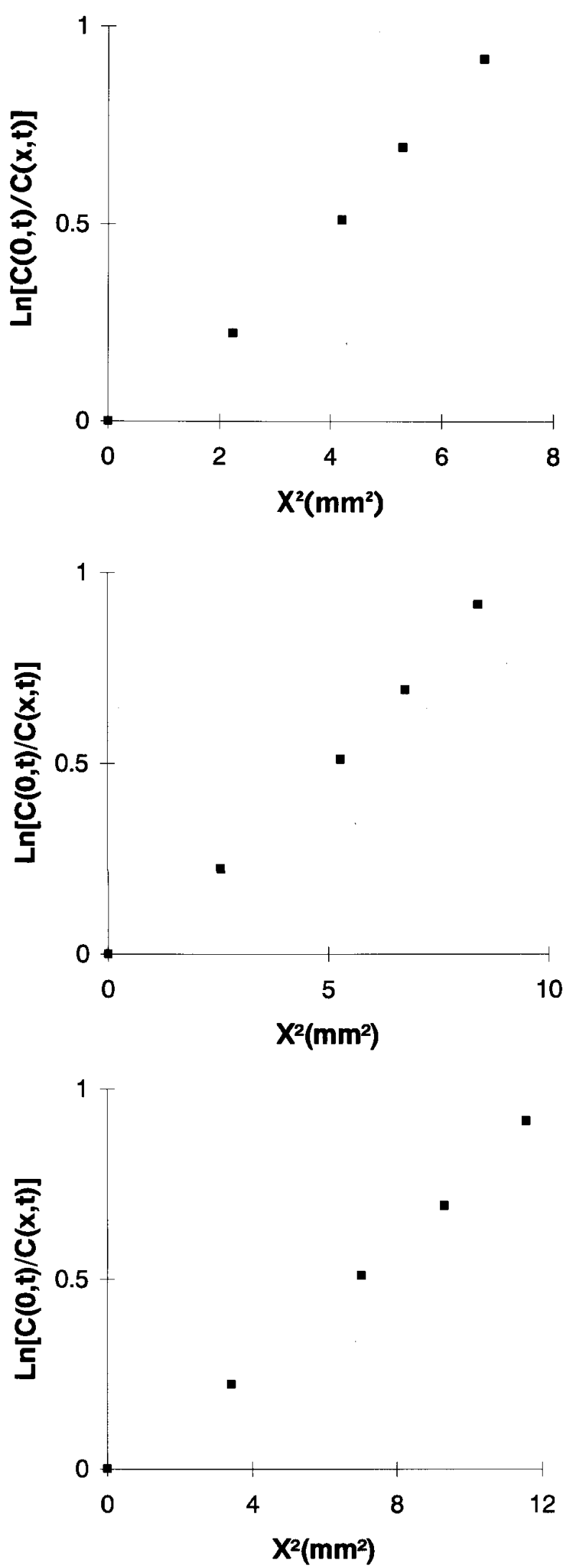

Figure 10. Plot of $\ln (C(0, t) / C(x, t))$ versus x2 of $\left(C_{6} H E T\right)$ at $85 \mathrm{C}(\mathrm{n} \mathrm{t} 2=72 \mathrm{~h})$. (a) in homeotropic orientation (red dye); (b) in planar orientation (red dye); (c) in planar orientation (yellow dye).
On the other hand $\Gamma$ the diffusion coefficients and the anisotropic ratios of polycatenar compounds are deduced from the pictures. First $\Gamma$ we consider the homeotropic orientation of mesophases $\Gamma$ we have obtained a circular geometry (Fig. 7a). As expected $\Gamma$ the anisotropy seems insignificant. Let us consider now the planar orientation $\Gamma$ we obtain an elliptical geometry (Fig. 7b). The measurement of the ellipse axe lengths allows to the anisotropic ratios. We conclude that the orientation quality is true. $\ln$ fact $\Gamma$ the symmetry of the elliptical diffusion (planar orientation) or the circular one (homeotropic orientation) is an evidence of the orientation quality in both directions. We present in table 2 the results for the used polycatenar compounds.

For the surface diffusion $\Gamma$ we plot $C(x, t)$ versus $x$ for three times ( 3 hours $\Gamma 5$ hours $\Gamma$ and 9 hours) for the case of $C_{5} H E T$ (Fig. 11aIbГ). We note that the concentration at the origin $C(O, t)$ decreases with the time $t$. Measuring the ratio $C\left(0, t_{i}\right) / C\left(O, t_{j}\right)$, we observe a good agreement between the experimental values with those predicted by the pre-exponential factor $C\left(0, t_{i}\right) / C\left(O, t_{j}\right)=\sqrt{t_{j} / t_{i}}$. We conclude that $\exp <\Gamma_{0}>\left(t_{j}-t_{i}\right) \approx 1$, then $<\Gamma_{0}>=0$. This means that the transverse diffusion can be neglected. The measurements of experimental and theoretical ratios are reported in table 3.

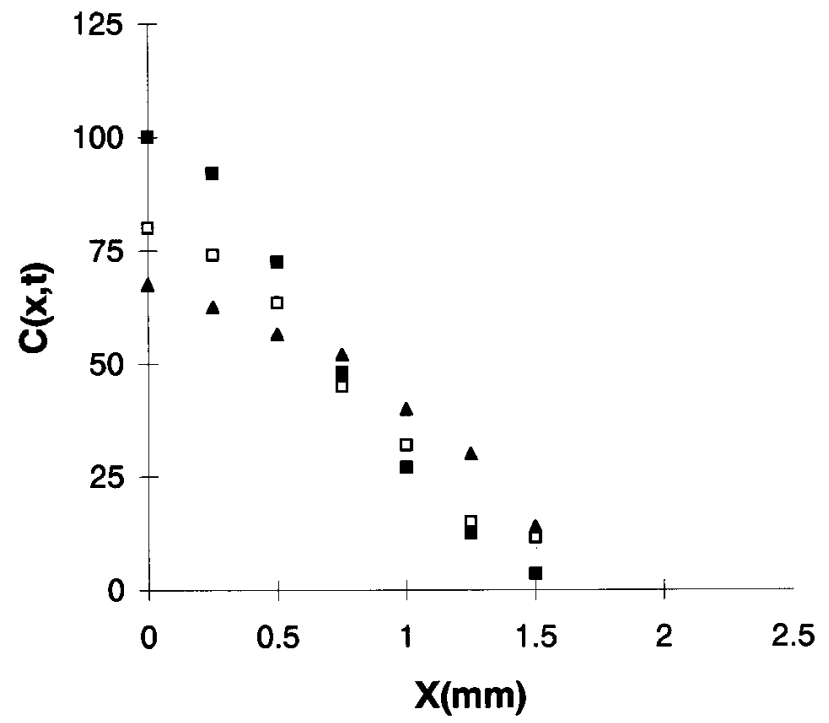

Figure 11. Plot of yellow dye concentration $\mathrm{C}(\mathrm{x}, \mathrm{t})$ versus position $\mathrm{x}$ of the $\left(C_{5} H E T\right)$ for three times: (a) $\mathrm{t} 1=3 \mathrm{~h}$; (b) $\mathrm{t} 2=5 \mathrm{~h} ;(\mathrm{c}) \mathrm{t} 3=9 \mathrm{~h}$. 
Table 1: Variation of the diffusion coefficients $\left(D_{\perp}\right) \Gamma\left(D_{\|}\right)$and the ratio in hexagonal columnar phases exhibited by some discotic compounds (d) the nature of aliphatic chains.

(a)

\begin{tabular}{llll}
\hline Coefficients & Impurities & $T\left({ }^{\circ} \mathrm{C}\right)=75$ & $T\left({ }^{\circ} \mathrm{C}\right)=85$ \\
\hline$D_{\perp} \times 10^{7} \mathrm{~cm}^{2} / \mathrm{s}$ & $\begin{array}{l}\text { 1-[4-(xylylazo)xylylazo]-2-Naphtol } \\
\text { 4-Phenylazophenol }\end{array}$ & 0.95 & 1.32 \\
\hline$D_{/ /} \times 10^{7} \mathrm{~cm}^{2} / \mathrm{s}$ & $\begin{array}{l}\text { 1-[4-(xylylazo)xylylazo]-2-Naphtol } \\
\text { 4-Phenylazophenol }\end{array}$ & $\begin{array}{l}1.48 \\
1.80\end{array}$ & 1.90 \\
& & & 2.71 \\
\hline$D_{/ /}$ & 1-[4-(xylylazo)xylylazo]-2-Naphtol & 1.56 & 1.39 \\
& 4-Phenylazophenol & 1.39 & 1.29 \\
& & & \\
\hline
\end{tabular}

(b)

\begin{tabular}{lllll}
\hline Coefficients & Impurities & $T\left({ }^{\circ} \mathrm{C}\right)=75$ & $T\left({ }^{\circ} \mathrm{C}\right)=85$ & $T\left({ }^{\circ} \mathrm{C}\right)=105$ \\
\hline$D_{\perp} \times 10^{7} \mathrm{~cm}^{2} / \mathrm{s}$ & 4-Phenylazophenol & 0.66 & 0.94 & 1.64 \\
\hline$D_{/ /} \times 10^{7} \mathrm{~cm}^{2} / \mathrm{s}$ & 4-Phenylazophenol & 1.20 & 1.49 & 2.55 \\
\hline$D_{/ /}$ & 4-Phenylazophenol & 1.88 & 1.58 & 1.50 \\
\hline$D_{\perp}$ & & & & \\
\hline
\end{tabular}

(c)

\begin{tabular}{lllll}
\hline Coefficients & Impurity & $C_{5} H E T$ & $C_{6} H E T$ & $C_{8} H E T$ \\
\hline$D_{\perp} \times 10^{7} \mathrm{~cm}^{2} / \mathrm{s}$ & 4-Phenylazophenol & 0.66 & 1.32 & 1.75 \\
\hline$D_{/ /} \times 10^{7} \mathrm{~cm}^{2} / \mathrm{s}$ & 4-Phenylazophenol & 1.20 & 1.84 & 1.93 \\
\hline$D_{/ /}$ & 4-Phenylazophenol & 1.88 & 1.39 & 1.10 \\
\hline$D_{\perp}$ & & & & \\
\hline
\end{tabular}




\begin{tabular}{llll}
\hline Coefficients & Impurity & $C_{8} H E T$ & $C_{1} 1 H E T$ \\
\hline$D_{\perp} \times 10^{7} \mathrm{~cm}^{2} / \mathrm{s}$ & 1-[4-(xylylazo)xylylazo]-2-Naphtol & 1.24 & 4.61 \\
\hline$D_{/ /} \times 10^{7} \mathrm{~cm}^{2} / \mathrm{s}$ & 1-[4-(xylylazo)xylylazo]-2-Naphtol & 1.61 & 10.96 \\
\hline$D_{/ /}$ & 1-[4-(xylylazo)xylylazo]-2-Naphtol & 1.30 & 2.40 \\
\hline$D_{\perp}$ & & & \\
\hline
\end{tabular}

Table 2: Measurements of the diffusion coefficients $\left(D_{\perp}\right) \cdot \Gamma\left(D_{\|}\right)$and the ratio in hexagonal columnar phases exhibited by some polycatenar mesogens.

\begin{tabular}{llllc}
\hline Coefficients & Impurity & $\begin{array}{l}\text { Heacatenar } \\
\left(C_{12}\right)\end{array}$ & $\begin{array}{l}\text { Tetracatenar } \\
\left(C_{10}\right)\end{array}$ & $\begin{array}{l}\text { Tetracatenar } \\
\left(C_{14}\right)\end{array}$ \\
\hline$D_{\perp} \times 10^{7} \mathrm{~cm}^{2} / \mathrm{s}$ & 1 -[4-(xylylazo)xylylazo]-2-Naphtol & 1.52 & 1.00 & 2.54 \\
\hline$D_{/ /} \times 10^{7} \mathrm{~cm}^{2} / \mathrm{s}$ & 1 -[4-(xylylazo)xylylazo]-2-Naphtol & 3.80 & 2.28 & 3.80 \\
\hline$D_{/ /}$ & 1 -[4-(xylylazo)xylylazo]-2-Naphtol & 2.02 & 2.28 & 1.86 \\
\hline
\end{tabular}

Table 3: Measurements of the experimental and theoretical ratio $D_{\|} \Gamma D_{\perp}$.

\begin{tabular}{llll}
\hline Ratio & $\mathrm{t}=3$ hours & $\mathrm{t}=5$ hours & $\mathrm{t}=9$ hours \\
\hline$\frac{C\left(0, t_{i}\right)}{C\left(0, t_{j}\right)}(\exp )$ & 1.26 & 1.30 & 1.60 \\
\hline$\frac{C\left(0, t_{i}\right)}{C\left(0, t_{j}\right)}(t h e)$ & 1.29 & 1.34 & 1.73 \\
\hline
\end{tabular}

Table 4: (a) Measurements of the surface diffusion coefficient (b) Comparison of surface diffusion coefficients with the bulk ones in the $\left(C_{5} H E T\right)$.

(a)

\begin{tabular}{lllll}
\hline Coefficients & Impurity & $T\left({ }^{\circ} \mathrm{C}\right)=75$ & $T\left({ }^{\circ} \mathrm{C}\right)=85$ & $T\left({ }^{\circ} \mathrm{C}\right)=105$ \\
\hline$D_{s} \times 10^{7} \mathrm{~cm}^{2} / \mathrm{s}$ & 4-Phenylazophenol & 1.75 & 2.30 & 3.20 \\
\hline
\end{tabular}




\begin{tabular}{lllll}
\hline$T\left({ }^{\circ} \mathrm{C}\right)$ & $D_{s} \times 10^{7} \mathrm{~cm}^{2} / \mathrm{s}$ & $D_{v / /} \times 10^{7} \mathrm{~cm}^{2} / \mathrm{s}$ & $D_{v \perp} \times 10^{7} \mathrm{~cm}^{2} / \mathrm{s}$ & $\frac{D_{s}}{D_{b}}$ \\
\hline 75 & 1.75 & 1.20 & 0.66 & 1.46 \\
\hline 85 & 2.30 & 1.49 & 0.94 & 1.54 \\
\hline 105 & 3.20 & 2.55 & 1.64 & 1.41 \\
\hline
\end{tabular}

Furthermore $\Gamma$ we have measured the $D_{s}$ values for the following temperatures $T_{1}=75^{\circ} \mathrm{C}, \quad T_{2}=85^{\circ} \mathrm{C}$ and $T_{3}=105^{\circ} \mathrm{C}$ (Table $4 \mathrm{a}$ ). We note that the $D_{s}$ values are nearer than the bulk one in planar orientation (Table 4b).

From the measured values $\Gamma$ we can make the following remarks:

(i) The matter diffusion in hexagonal columnar mesophases exhibited by discotic and polycatenar compounds is anisotropic whatever the dye size or the nature of the sample. The ratio $r=D_{\|} / D_{\perp}$ is always superior to 1 . This means that the diffusion is quicker along the columns than perpendicularly to them. This result seems in good agreement with previous experimental and theoretical studies [4I11] which allows to determine the structure of columnar discotic liquid crystals $\Gamma$ liquid-like order along the column direction and solid-like order perpendicularly to it. The anisotropy decreases when the temperature increases. Indeed $\Gamma$ when the temperature comes up to liquid phase $\Gamma$ the fluidity of mesophase increases and the system tends toward an isotropic state.

It is interesting to compare the polycatenar results to the discotic ones. Table 5 gives the anisotropic ratios and shows that for the columnar polycatenars $\Gamma$ we obtain $1.86 \leq D_{\|} / D_{\perp} \leq 2.28$ whereas this ratio varies as $1.10 \leq D_{\|} / D_{\perp} \leq 1.88$ in the columnar discotic phases. We notice that the polycatenar anisotropic ratios are larger than the corresponding ones in discotic mesophases. This is probably due to the columnar structure in both cases. Indeed $\Gamma$ in the discotic compounds Tthe disk-like molecules which pile up are staked one after one and arranged in a two dimensional network $\Gamma$ whereas in the polycatenar compounds $\Gamma$ the average number of molecules in a slice of column is three for phasmids and four for biforked mesogens.

Table 5: Comparison of $D_{\|} / D_{\perp}$. ratios measured in discotic compounds with polycatenar ones.

\begin{tabular}{lllllll}
\hline ratio & \multicolumn{3}{c}{ Hexa-n-alkoxytriphenylenes } & & \multicolumn{2}{c}{ Polycatenars } \\
\hline & $C_{5} H E T$ & $C_{6} H E T$ & $C_{8} H E T$ & $\operatorname{Tetra}\left(C_{10}\right)$ & $\operatorname{Hexa}\left(C_{12}\right)$ & $\operatorname{Tetra}\left(C_{14}\right)$ \\
\hline$\frac{D_{/ /}}{D_{\perp}}$ & 1.88 & 1.39 & 1.10 & 2.28 & 2.02 & 1.86 \\
\hline
\end{tabular}

In addition $\Gamma$ the diffusion anisotropic ratio is larger for the $C_{11} H A T$ compound $(r=2,4)$ than the $C_{8} H E T$ one $(r=1,3)$. This is probably due to the difference in length and type of the aliphatic chains bound to the triphenylene cores (ether chain: $-0-C_{8} H_{17}$ and ester one: $\left.-O C O-C_{11} H_{23}\right)$. In fact $\Gamma$ the inter-columnar distances for the two mesophases increase from $2 \mathrm{I} 22 \mathrm{~nm}$ for the $C_{8} H E T$ to $2.66 \mathrm{~nm}$ for the $C_{11} H A T$. More $\mathrm{Lun}-$ 
like ether $\Gamma$ the ester chains of the $C_{11} H A T$ are not in the plane of disk $\Gamma$ but the oxygen atoms of carboxylic group are lying on both sides of the disk plane.

(ii) A comparison of the surface diffusion results with to bulk ones in planar orientation leads a ratio $D_{s} / D_{b} \approx 1.5$ (Table $4 \mathrm{~b}$ ). This result can be explained on the basis of point defects $\Gamma$ i.e. the existence of the column extremities Ttaking into account of the existence of fault lines. The corresponding structure is perturbed only in a plane where the columns jump from one site to the next. The columnar sample is perfect immediately above and below this plane. These lines can taper into point defects which are column extremities [11]. Using the atomic theory $\Gamma$ the diffusion coefficient $\mathrm{D}$ is given by $[56]$ :

$$
D=\frac{1}{n} \Gamma \lambda^{2}
$$

Where $n, \Gamma$ and $\lambda$ represent respectively the number of sites the jump frequency from one site to the next and the mean free path.

Given that the impurity diffusion takes place into the mesh of aliphatic chains surrounding the columns $\Gamma$ we acknowledge that the impurity can jump to four sites in surface diffusion (Fig. 12a) and to six sites in the bulk one (Fig. 12b). Then $\Gamma D_{s}=\Gamma \lambda^{2} / 4$ and $D_{b}=\Gamma \lambda^{2} / 6$. This allows $D_{S} / D_{b}=1.5$. We observe a good agreement between the experimental and the theoretical values.

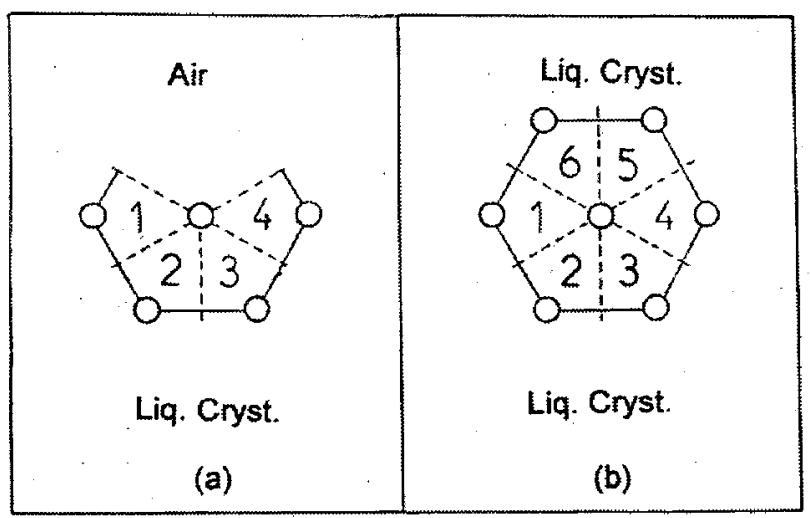

Figure 12. Schematic representation of the impurity jump in sites: (a) in surface diffusion (four sites); (b) in bulk diffusion (six sites).

(iii) For all the studied discotic $\left(C_{5}, C_{6}, C_{8}\right)$ and polycatenar $\left(C_{10}, C_{12}, C_{14}\right)$ compounds $\Gamma$ the diffusion coefficients $D_{\|} / D_{\perp}$ increase with the length of aliphatic chains. Assuming that the diffusion impurity molecules take place in the volume occupied by these chains $\Gamma$ we can explain this result by the width of paraffinic crown surrounding the core. From the parameter a of the hexagonal lattice [4] for instance $\Gamma$ we have estimated this crown width for the discotic compounds as about $1.2 \mathrm{~nm}\left(C_{5} H E T\right)$ and $1.6 \mathrm{~nm}\left(C_{8} H E T\right)$. A comparison of the $D_{\|} / D_{\perp}$ ratio in discotic and polycatenar compounds (Table 5) shows that this ratio decreases with the length of aliphatic chains in both cases. This is due to the increasing of the crown width. Furthermore $\Gamma$ this ratio is greater for the polycatenar compounds. This effect can be explained by the stacking disorder of molecules in columns.

For a comparison between the diffusion along the columns and perpendicularly to them $\Gamma$ in discotic and polycatenar compounds $\Gamma$ we calculate the average ratio

$$
\frac{\sum D_{\text {Polycatenars }}}{\sum D_{\text {Discotics }}}
$$

and find:

$$
\frac{\sum D_{\perp \text { Polycatenars }}}{\sum D_{\perp \text { Discotics }}} \approx 1, \quad \frac{\sum D_{\| \text {Polycatenars }}}{\sum D_{\| \text {Discotics }}} \approx 2,
$$

The first ratio $(\approx 1)$ shows that the diffusion perpendicularly to columns is comparable in both columnar mesophases. If we assume that the molecules are aligned along the radius of a circular column we obtain a very dense paraffinic zone [56]. As in the discotic case $\Gamma$ the matter diffusion in polycatenar mesogens bypasses the central part of columns and takes place in the paraffinic crown.

The second ratio $(\approx 2)$ shows that the diffusion along the columns is quicker in the polycatenar mesophases. This effect can be explained by the increasing of the lateral chain lengths $(\mathrm{n}=5 \Gamma 6 \Gamma 8$ for the discotics and $n=10 \Gamma 12 \Gamma 14$ for the polycatenars). Furthermore $\Gamma$ using a chemical affinity $\Gamma$ we can still justify this effect by the impurity polarity (presence of phenol function in Red Oil 0) which is more soluble in polycatenar compounds containing a polar group ($\mathrm{CH}=\mathrm{N}-$ ). This effect may be explained too as a more relative disordered disposition of the molecules along a column in comparison with the long range order observed in discotic columns.

(iiii) The diffusion is faster for the Phenylazophenol (yellow dye) of which the molecular size is smaller than the 1-[4-(xylylazo)xylylazo]-2-Naphtol (Red Oil OГ red dye). Furthermore $\Gamma$ the ratios of diffusion coefficients vary approximately in the same proportion. 
For the $C_{6} H E T$, for instance $\Gamma$ at the same temperature $\left(T=85^{\circ} \mathrm{C}\right)$ these ratios are estimated as follow:

$$
\frac{\sum D_{\perp \text { red dye }}}{\sum D_{\perp \text { reddye }}} \approx \frac{\sum D_{\| \text {yellow dye }}}{\sum D_{\| \text {yellow dye }}} \approx 0.7
$$

This result seems in agreement with a theoretical ratio which takes account of the diameter $l$ of self diffused molecules. lndeed $\Gamma$ using at the same temperatureГa relationship for the smectic $\Gamma$ nematic and isotropic phases $[58] \Gamma D \propto 1 / l$ we obtain:

$$
\frac{\sum D_{\perp \text { reddye }}}{\sum D_{\perp \text { reddye }}} \approx \frac{\sum D_{\| \text {yellow dye }}}{\sum D_{\| \text {yellow dye }}} \approx 0.8
$$

Then $\Gamma$ the experimental ratio is comparable to the theoretical one. This means that the size effect of the diffused dye has the same behaviour in the mesophases and in the isotropic phases.

\section{b) Activation energy}

It is established that the relationship between the activation energy $\mathrm{E}$ and the diffusion coefficient $D$ is given by the Arrhenius equation:

$$
D=D_{0} \exp \left(-\frac{E}{R T}\right)
$$

where $E$ is the activation energy and $R \Gamma$ the gas constant.

In the solid state $\Gamma E$ is interpreted as the potential barrier over which the diffusing particle has to jump from one lattice position to the next. In the liquid state $\Gamma$ it is assumed that a diffusing particle is an activated state during the jump. This concept leads to a temperature dependence of the coefficient $D_{0}$. This effect $\Gamma$ however $\Gamma$ is so weak that the Arrhenius law is still a very good approximation even in a liquid state. For liquid crystals which are in an intermediate state between solid and liquid $\Gamma$ the argument is still true and the Arrhenius law may be used for the diffusion data.

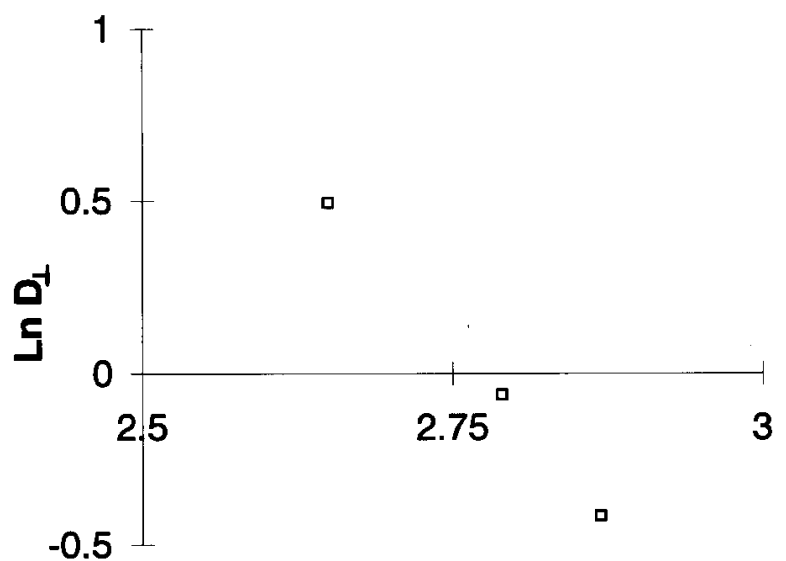

$1 / \mathrm{T} \cdot 10^{3}(\mathrm{~K}-1)$

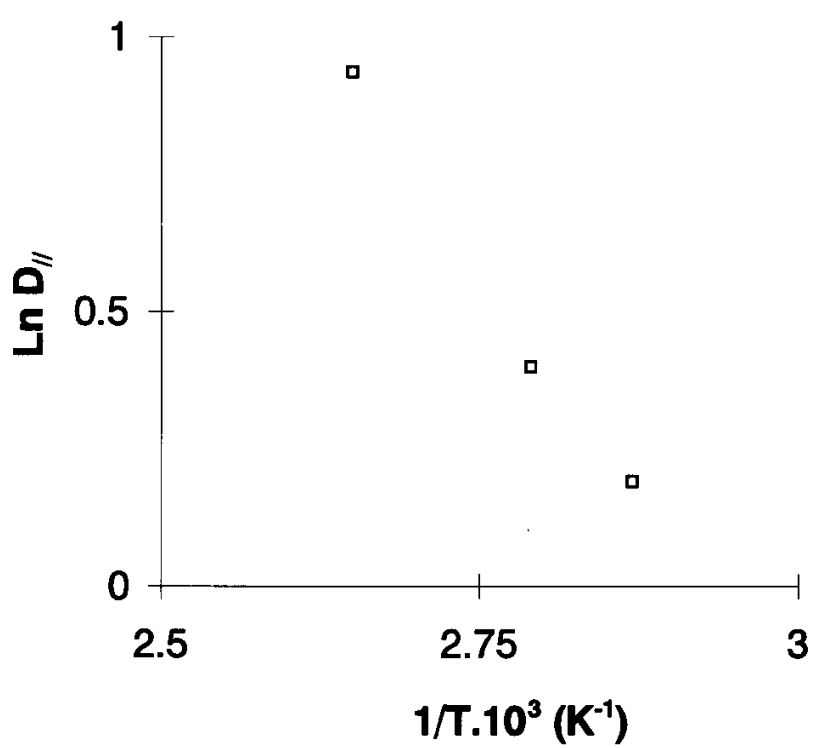

Figure 13. Plot of $\mathrm{LnD}_{\perp}$ and $\mathrm{LnD}_{\|} /$versus $\mathrm{T}-1$ in the $\left(C_{5} H E T\right)$ (a) in homeotropic orientation (b) in planar orientation.

To determine the activation energy in the two geometries of the sample orientation $\Gamma$ for the $C_{5} H E T$ for instance $\Gamma$ we plot the logarithms of diffusion coefficients $\ln D_{\perp}$ and $\ln D_{\|}$versus reciprocal temperature $\frac{1}{T}$ (Fig. 13aIb). We observe a linear dependence as expected from the Arrhenius equation. From the slope we can deduce:

$$
\begin{gathered}
E_{\perp}=34.5 \mathrm{~kJ} . \mathrm{mol}^{-1} \\
E_{\|}=30.3 \mathrm{~kJ} . \mathrm{mol}^{-1} \\
\Delta E \approx 5 \mathrm{~kJ} . \mathrm{mol}^{-1}
\end{gathered}
$$

We note that these values are similar i.e. $\Gamma$ unlike the diffusion coefficients which present an anisotropic ratio the activation energies $E_{\|}$and $E_{\perp}$ of particles seem 
independent from the diffusion geometry. Indeed $\Gamma$ seeing that the dye diffusion takes place into the volume occupied by aliphatic chains $\Gamma$ whatever the orientation geometry the matter diffusion throught the mesophase requires approximately the same avtivation energy.

\section{Comparison with nematic and smectic mesophases}

A comparison of the dyes diffusion in thermotropic columnar mesophases exhibited by disk-like and polycatenar compounds to some nematic ones shows a similar ratio $D_{\|} / D_{\perp}$. For exemple $\Gamma$ the diffusion at room temperature of some impurities such the tetramethylsilane (TMS) $\Gamma$ the methyl-red (MR) or the nitrosodimethylaniline (NMA) into nematic mesophases exhibited by the p-metoxybenzylidene-p'-n-butylaniline (MBBA) or by the Licristal $\left(L C_{4}\right)$ gives anisotropic ratios $D_{\|} / D_{\perp}$ which vary from 1.03 to 1.70 (Table 6 ). In addition $\Gamma$ the activation energy in nematic phases shows $\Gamma$ as the columnar ones $\Gamma$ an insignificant anisotropy $\left(0.98<E_{\|} / E_{\perp}<1.06\right)$. This means that the diffusion features in columnar and nematic mesophases are com- parable. This result seems to be in agreement with a recent study of diffusivity by Light Beating Spectroscopy in thermotropic nematic mesophases exhibited by disklike mesogens [59]. It was shown that the nematic order can be manifest in the packing of a few molecules (15 to 25) to form relatively short columns of a diameter of an individual disk. Each column would be equivalent to an individuel rod in classical nematic liquid crystals. Furthermore $\Gamma$ the ratio $E_{\|} / E_{\perp}$ is often used to characterize the anisotropy of diffusion in smectic mesophases. Previous studies [14] have shown that in the smectic mesophases this ratio is $\gg 1$. These results are in qualitative agreement with the structural features of theses mesophases which consist of liquid layer. Thus Tinside the layers the diffusion is liquid-like with a lower activation energy as in the solid-like jump process from layer to layer. Unlike the columnar mesophases where the impurity diffusion takes place into the volume occupied by aliphatic chains whatever the orientation geometries (diffusion parallel to the columns or perpendicularly to them) $\Gamma$ the matter diffusion requires approximately the same activation energy. So $\Gamma$ we find $E_{\|} / E_{\perp} \approx 1$.

Table 6: Comparison of impurity diffusion coefficients in columnar phases to nematic and smectic ones.

\begin{tabular}{lllllll}
\hline Phase & Mesogenic Compounds & Impurities & $T\left({ }^{\circ} C\right)$ & $\frac{D_{/ /}}{D_{\perp}}$ & $\frac{E_{/ /}}{E_{\perp}}$ & References \\
\hline Nematic & MBAA & $4 \%$ TMS & 22 & 1.03 & 1.04 & {$[14]$} \\
& MBBA & $8 \%$ TMS & 22 & 1.00 & 1.04 & {$[14]$} \\
& MBBA & NMR & 22 & 1.70 & - & {$[17]$} \\
& MBBA & MR & 22 & 1.60 & - & {$[17]$} \\
& LC4 & $2 \%$ TMS & - & 1.26 & 0.98 & {$[14]$} \\
& LC4 & 10\% TMS & - & 1.09 & 1.04 & {$[14]$} \\
\hline Hexagonal Columnar & $C_{5} H E T$ & Yellow dye & 75 & 1.88 & $\approx 1$ & {$[23]$} \\
& $C_{6} H E T$ & Yellow dye & 75 & 1.39 & - & {$[23]$} \\
& & Red Oil O & 75 & 1.56 & - & {$[23]$} \\
& $C_{8} H E T$ & Red Oil O & 75 & 1.30 & - & {$[24]$} \\
& \multirow{2}{*}{$C_{11} H$ AT } & Yellow dye & 75 & 1.10 & - & {$[24]$} \\
& & Red Oil O & 90 & 2.40 & - & {$[24]$} \\
& & Yellow dye & 115 & 2.20 & - & {$[24]$} \\
\hline Hexagonal phasmidic & Tetracatenar $\left(C_{10}\right)$ & Red Oil O & 75 & 2.28 & - & {$[26]$} \\
& Hexacatenar $\left(C_{12}\right)$ & Red Oil O & 75 & 2.02 & - & {$[26]$} \\
& Tetracatenar $\left(C_{14}\right)$ & Red Oil O & 75 & 1.86 & - & {$[26]$} \\
\hline
\end{tabular}




\section{Conclusion}

We have shown that optical absorption and densitometry techniques can be used to determine the diffusion behaviour of dyes in thermotropic hexagonal columnar mesophases exhibited by discotic and polycatenar compounds. Whatever the orientation geometries (homeotropic and planar) $\Gamma$ the nature of the sample (bulk and thread) $\Gamma$ the molecular form (disk-like and rod-like) $\Gamma$ we have measured the diffusion coefficients $D_{\|}$and $D_{\perp}$ (along the columns or perpendicularly to them) and the ratios $D_{\|} / D_{\perp}$. The experimental values show an anisotropic diffusion in both cases.

The anisotropic behaviour becomes less pronounced when the temperature increases. In addition $\Gamma$ whatever the dye size $\Gamma$ the diffusion of impurities into the mesophases takes place in the volume occupied by the aliphatic chains. Furthermore $\mathrm{\Gamma}$ a comparison of results shows that the diffusion along the columns is quicker in phasmidic compounds than in discotic ones. This is probably due to a relative disordered arrangement of the molecules along the columns of phasmidic mesophases in comparison with the short order observed in discotic columns. We conclude that the molecular structure of polycatenar compounds holds in an intermediate position between the mesogenic disks and mesogenic rods.

The anisotropic behaviour of matter diffusion in columnar discotic mesophases 5 studied also by mirage effect techniques confirms the obtained effects of impurity size length and type of branched alphatic chains.

In addition $\Gamma$ the symmetry of diffusion pictures of the concentration profiles (circular for the homeotropic orientation and elliptic for the planar one) proves the average macroscopic orientation.

The temperature dependence of $D_{\|}$and $D_{\perp}$ can be described by the Arrhenius Law. The activation energies $E_{\|}$and $E_{\perp}$ are about equal to each other.

A comparison of our results to some others shows that $\Gamma$ as in nematics and smectics $\Gamma$ the diffusion in thermotropic columnar liquid crystals (discotics and polycatenars) is anisotropic. However $\Gamma$ the activation energy ratio $E_{\|} / E_{\perp}$ is insignificant as in the nematic phase. So Tunlike in smectic mesophases $\Gamma$ this ratio can not be used to characterize the columnar mesophase anisotropy.

\section{References}

[1] F.C. Franck, S. Chandrasekhar, J. Phys. 40, 1285 (1980).

[2] P. Oswald, M. Kléman, J. Phys. 42, 1461 (1981).

[3] P. Oswald, M. Kléman, J. Phys. 49, 1083 (1988).

[4] A. M. Levelut, J. Chem. Phys. 80, 149 (1983).

[5] M. Cagnon, M. Gharbia and G. Durand, Phys. Rev. Lett. 53, 938 (1984).

[6] P. Oswald, J. Malthêute, P. Pelcé, J. Phys. (France), 50, 2121 (1989).

[7] S. Chandrasekhar, G.S. Ranganath, Rep. Prog. Phys. 53, 37 (1990).

[8] M. Gharbia, A. Gharbi, M. Cagnon and G. Durand; J. Phys. (France), 51, 1355 (1990).

[9] J. Prost, Liq. Cryst., 8, 1285 (1990).

[10] M. Gharbia, T. Othman, A. Gharbi, C. Destrade, G. Durand, Phys. Rev. Lett. 68, 13 (1992).

[11] P.G. de Gennes, J. Prost, The Physics of Liquid Crystals, Oxford Univ. Press (1993).

[12] L. Sallen, P. Oswald, J.C. Guminard and J. Malthête, J. Phys.II (France), 5, 937 (1995).

[13] T. Svedberg, Kolloidzeitschrift, 22, 68 (1918).

[14] G.J. Krüger, Phys. Rep. 82, N. 4229 (1982).

[15] C.K. Yan, A.G. Fredrickson, Mol. Cryst. and Liq. Cryst. 12, 13 (1970).

[16] E. Rondalez, Sol. State Commun. 14, 815 (1974).

[17] H. Hakemi, M.M. Labes, J. Chem. Phys. 63, 3708 (1975).

[18] C.G. Wade, Ann. Rev. Chem. 2847 (1977).

[19] I. Zupancic, J. Pirs, M. Luzar, R. Blinc and J.W. Doane, Sol. State Commun. 15, 227 (1974).

[20] G.J. Krüger, H. Spiesecke, R. Van Stenwinkel and F. Noack, Mol. Cryst. and Liq. Cryst. 40, 103 (1977).

[21] J. Topler, B. Alfred and T. Springer, Mol. Cryst. and Liq. Cryst. 26, 297 (1974).

[22] F. Volino, A.J. Dianoux and A. Heidermann, J. Phys. Lett. 40, L-583 (1979).

[23] M. Daoud, M. Gharbia and A. Gharbi; J. Phys. Il (France), 4, 989 (1994).

[24] M. Daoud, G. Louis, X. Quulin, M. Gharbia, A. Gharbi and P. Peretti, Liq. Cryst. 19, 833 (1995).

[25] M. Daoud, M. Gharbia, A. Gharbi and C. Destrade, Surf. Sci. 349, L174 (1996). 
[26] M. Daoud, K. Rais, M. Gharbia, A. Gharbi and H.T. Nguyen, submitted to Liq. Cryst. (1998).

[27] P. G. de Gennes, The Physics of Liquid Crystals, Oxford Univ. Press (1994).

[28] S. Chandrasekhar, B.K. Sadashiva and K.A. Sureh, Pranama, 9, 471 (1977).

[29] J.C. Dubois, Ann. Phys. N.Y. 3, 13 (1978).

[30] C. Destrade, M.C. Bernaud, H. Gasparoux, A.M. Levelut and H.T. Nguyen, Proc. lnt. Conf Liq. Cryst. (Bangalore), 1980a (1979).

[31] C. Destrade, H.T. Nguyen, H. Gasparoux, J. Malthête and A.M. Levelut, Mol. Cryst. and Liq. Cryst. 71, 111 (1981).

[32] M. Dvolaitzzky, J. Billard, Mol. Cryst. and Liq. Cryst. 64, 247 (1981).

[33] C. Destrade, H. Gasparoux, P. Foucher, H.T. Nguyen, J. Malthête and J. Jacques, J. Chem. Phys. 80, 137 (1983).

[34] P. Foucher, C. Destrade, H.T. Nguyen, J. Malthête and A.M. Levelut, Mol. Cryst. and Liq. Cryst. 108, 219 (1984).

[35] J. Malthête, C. Destrade, H.T. Nguyen and J. Jacques, Mol. Cryst. and Liq. Cryst. 66, 115 (1981).

[36] J. Malthête, J. Jacques, H.T. Nguyen and C. Destrade, Nature 298, 46 (1982).

[37] E. Fontes, P.A. Heiney, W.H. de Jeu, Phys. Rev. Lett. 61, 1202 (1988).

[38] K.D. Kamien, D.R. Nelson, Phys. Rev. Lett. 74, 2499 (1995).

[39] K.D. Kamien, D.R. Nelson, Phys. Rev. E53, 650 (1996).

[40] M. Hebert, A. Caillu, Phys. Rev. E53, 1714 (1996).

[41] J. Malthête, A.M. Levelut, H.T. Nguyen, J. Phys. Lett. 46, L 875 (1985).

[42] H.T. Nguyen, C. Destrade, A.M. Levelut, J. Malthête, J. Phys. 47, 553 (1986).
[43] C. Destrade, H.T. Nguyen, A. Roubineau and A.M. Levelut, Mol. Cryst. and Liq. Cryst. 159, 163 (1988).

[44] J. Malthête, H.T. Nguyen and C. Destrade, Liq. Cryst. 13, 171 (1993)

[45] J. Malthête, A. Collet and A.M. Levelut, Liq. Cryst. 5 , 129 (1989).

[46] H.T. Nguyen, C. Destrade and J. Malthête, Adv. Mater. 9, 375 (1997).

[47] H. Strzelecka, C. Jallabert, M. Veber, P. Davidson and A. M. Levelut, Liq. Cryst. 161, 395 (1988).

[48] M.Veber, P. Sotta, P. Davidson, A.M. Levelut, C. Jallabert and H. Strzelecka, J. Phys. (France), 51, 1283 (1990).

[49] A. Zinsou, M. Veber, H. Strzelecka, C. Jallabert and A.M. Levelut, Liq. Cryst. 17, 513 (1994).

[50] V. Luzzari, Biological Membranes, Ed. Chapman (Acad. Press, London) 1968.

[51] P. Ekwall, Adv. Liq. Cryst. 1, 1 (1975).

[52] A.E. Soulios, A. Luzarti, Acta Cryst. 14, 278 (1961).

[53] C. Piechocki, J. Simon, A. Skoulios, D. Guillon and P. Weber, J. Am. Chem. Soc. 104, 5245 (1982).

[54] D.M. Van Winkle, N.A. Clark, J. Phys. Lett. 48, 1407 (1982).

[55] C.R. Safinya, K.S. Liang, W.A. Varady N.A. Clark and G. Anderson, J. Phys. Lett. 53, 1172 (1984).

[56] J. Phillibert, Diffusion et transport de matiere dans les solides, Ed. de Phys. (1985).

[57] O. Prodi, J. Phys. Lett. 37, L143 (1976).

[58] A.M. Levelut, J. Malthête, C. Destrade and H.T. Nguyen, Liq. Cryst. 2, 877 (1987).

[59] T. Othman, M. M. Jebari, A. Gharbi and G. Durand, Mol. Cryst. and Liq. Cryst. 281, 145 (1996). 